\title{
BMJ Open Multilevel mobile health approach to improve cardiovascular health in resource-limited communities with Step It Up: a randomised controlled trial protocol targeting physical activity
}

To cite: Tamura $\mathrm{K}$, Vijayakumar NP, Troendle JF, et al. Multilevel mobile health approach to improve cardiovascular health in resource-limited communities with Step It Up: a randomised controlled trial protocol targeting physical activity. BMJ Open 2020;10:e040702. doi:10.1136/ bmjopen-2020-040702

- Prepublication history and additional material for this paper is available online. To view these files, please visit the journal online (http://dx.doi.org/10. 1136/bmjopen-2020-040702)

$\mathrm{KT}$ and NPV are joint first authors.

Received 22 May 2020 Revised 09 September 2020 Accepted 15 November 2020

Check for updates

(c) Author(s) (or their employer(s)) 2020. Re-use permitted under CC BY-NC. No commercial re-use. See rights and permissions. Published by BMJ.

For numbered affiliations see end of article.

Correspondence to Dr Tiffany M Powell-Wiley; tiffany.powell@nih.gov

\section{ABSTRACT}

Introduction Although physical activity $(\mathrm{PA})$ reduces cardiovascular disease (CVD) risk, physical inactivity remains a pressing public health concern, especially among African American (AA) women in the USA. PA interventions focused on AA women living in resourcelimited communities with scarce PA infrastructure are needed. Mobile health (mHealth) technology can increase access to PA interventions. We describe the development of a clinical protocol for a multilevel, community-based, mHealth PA intervention for AA women.

Methods and analysis An mHealth intervention targeting AA women living in resource-limited Washington, DC communities was developed based on the socioecological framework for PA. Over 6 months, we will use a Sequential Multi-Assignment, Randomized Trial approach to compare the effects on PA of location-based remote messaging (named 'tailored-to-place') to standard remote messaging in an mHealth intervention. Participants will be randomised to a remote messaging intervention for 3 months, at which point the intervention strategy will adapt based on individuals' PA levels. Those who do not meet the PA goal will be rerandomised to more intensive treatment. Participants will be followed for another 3 months to determine the contribution of each mHealth intervention to PA level. This protocol will use novel statistical approaches to account for the adaptive strategy. Finally, effects of PA changes on CVD risk biomarkers will be characterised.

Ethics and dissemination This protocol has been developed in partnership with a Washington, DC-area community advisory board to ensure feasibility and acceptability to community members. The National Institutes of Health Intramural IRB approved this research and the National Heart, Lung, and Blood Institute provided funding. Once published, results of this work will be disseminated to community members through presentations at community advisory board meetings and our quarterly newsletter.

\section{Strengths and limitations of this study}

- Community partnerships and a strong foundation of preliminary community-based participatory research studies establish trust and collaboration with the community, which are essential for acceptance and success of the community-based physical activity intervention

- This multilevel, technology-enabled approach to increase physical activity (PA) targets both individual motivations and barriers as well as neighbourhood resource utilisation.

- As an adaptive behavioural intervention, PA goals are determined based on each participants' individual baseline and adapt over time according to participants' response.

- This study assesses both clinical measures (body mass index, blood pressure, lipid profile, glucose levels, smoking status) and biological cardiovascular health mediators (metabolic, lipid and inflammatory pathways).

- The statistical power is based on the primary objective to evaluate effects of tailored-to-place and standard remote messaging on PA; analysis is underpowered to evaluate changes in cardiovascular risk markers, but exploratory analyses will evaluate potential trends.

Trial registration number NCT03288207.

\section{INTRODUCTION}

The ubiquity of smartphone use presents an opportunity to expand mobile health (mHealth)-based cardiovascular interventions, including those focused on physical 
Adapted Socio-Ecological Model for Step It Up Intervention

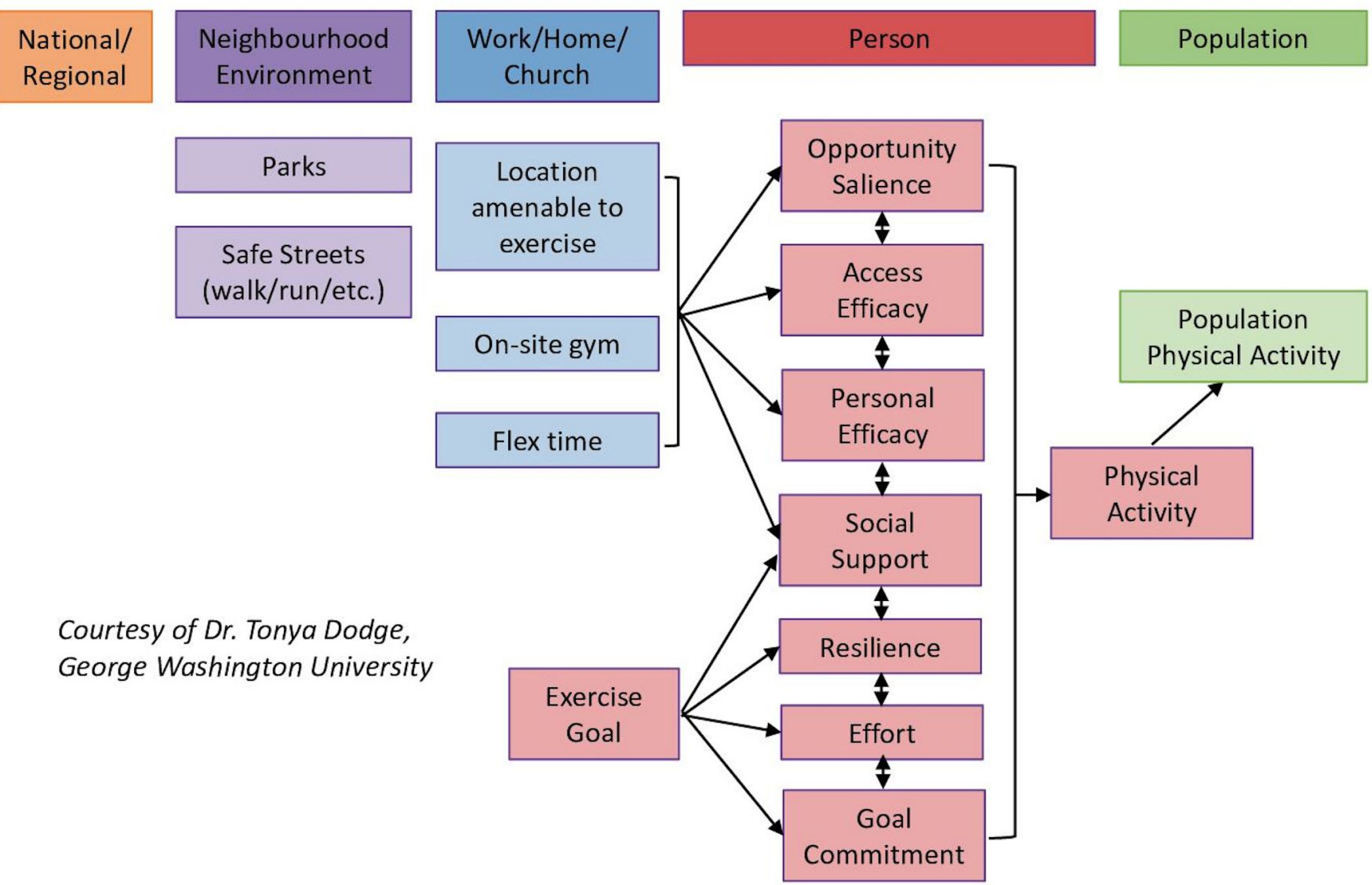

Figure 1 Adapted socioecological model that accounts for various factors affecting an individual's decision to engage in physical activity. Tailored-to-place messaging will focus on the neighbourhood environment and work/home/church levels of the socioecological model, while standard-remote messaging focuses on the person-level.

activity (PA), to broad community populations. ${ }^{1}$ A growing body of evidence suggests that mHealth technologyenabled interventions use text messaging, internet platforms or mobile applications (apps) to effectively promote PA, reduce sedentary behaviour and improve cardiovascular health. ${ }^{2-9}$ However, existing mHealth studies show conflicting results, are limited by small sample sizes, and focus on young, highly educated and high-income individuals. ${ }^{710}$ These samples do not reflect the populations at greatest risk for cardiovascular disease (CVD); research is needed to explore the utility of mHealth interventions within more diverse populations.

Among African American (AA) women, studies suggest that culturally appropriate interventions can effectively promote PA participation. ${ }^{11}{ }^{12}$ CVD disproportionately impacts AAs, and PA reduces CVD risk. ${ }^{13}$ AA women, who more often live in resource-limited neighbourhoods, have higher rates of sedentary time than other racial/ethnic groups. ${ }^{1415}$ According to the National Health Interview Survey 2018, 39.7\% of AA women meet PA guidelines, compared with $43.8 \%$ of Hispanic women and $54.7 \%$ of White women. ${ }^{16}$ Interventions to increase PA can be made more effective by targeting multiple levels of a socioecological framework for PA, including individual, interpersonal and neighbourhood environment-level factors (figure 1). ${ }^{17} 18 \mathrm{An}$ important contributing factor to PA is the local built environment, including sidewalks, well-maintained parks, recreation centres and perceived safety. ${ }^{19-23}$ Lower socioeconomic status neighbourhoods have fewer PA facilities, and are less conducive to obtaining adequate PA. ${ }^{24}$ To create an effective behavioural health intervention to reduce CVD disparities, it is critical to partner with the target community, and develop culturally appropriate strategies to navigate the local built environment. ${ }^{11} 12$

In this study, we introduce Step It Up, a communitybased PA intervention targeting at-risk AA women residing in resource-limited communities in the Washington, DC metropolitan area. Community-based participatory research principles were used to partner with local community leaders and design a 6-month mHealth intervention aimed at increasing participants' PA. We began with a health-and-needs assessment to determine feasibility of the intervention in our target population, followed by a pilot mHealth intervention tailored to the built environment of participants. ${ }^{25} 26$ The objective of this manuscript is to describe the study design and 
components, recruitment methods, proposed measurements, analysis plan, strengths and limitations.

\section{METHODS AND ANALYSIS \\ Development of the Step It Up intervention: preliminary studies \\ Community engagement to design culturally appropriate interventions}

Since beginning work in Washington, DC in 2013, our research team has sought to engage at-risk communities with limited access to resources for nutritious diet and PA. Washington, DC is divided into eight regions, called wards, for municipal purposes; our efforts focus on Washington, DC wards 5, 7, 8, and the contiguous Prince George's County, Maryland (MD). These wards have the highest burden of CVD and lowest income in Washington, DC, with gentrification often pushing the population into Prince George's County. ${ }^{27} 28$ These neighbourhoods also have fewer PA facilities compared with other parts the city, and $64 \%-91 \%$ of the population is AA. ${ }^{27} 2930$

We established the DC Cardiovascular Health and Obesity Collaborative (DC CHOC), a community advisory board which includes representatives with expertise and/or interest in cardiovascular health from faith-based and community organisations. ${ }^{31}$ DC CHOC provides feedback on the development and implementation of all community-oriented projects, including Step It Up. ${ }^{32-34}$ We worked with DC CHOC to first determine the feasibility of using wearable technology to measure and promote PA in the community through the Washington, DC Cardiovascular Health and Needs Assessment (DC CHNA). ${ }^{34}$ Ninety-eight percent of participants recruited for the DC CHNA lived in Washington, DC Wards 5, 7, 8 or Prince George's County, MD. Further, DC CHNA gave insight into setting an activity goal; evidence suggests that odds of all-cause mortality and metabolic syndrome are lowest in those taking $>7500$ steps/day, and 15000 steps/day may maximise health benefits. ${ }^{35-37}$ From observations in DC CHNA, a goal of 10000 steps/day was determined to be both feasible and beneficial. Incorporating communitybased participatory research methods has helped to ensure our interventions are culturally appropriate and acceptable, increasing the likelihood of adoption.

\section{Iterative user-centred development of Step It Up app}

To optimise the app's ability to facilitate improved health behaviours, end-user feedback was solicited from community focus groups (FGs) throughout the app development process. ${ }^{38}$ We worked with an app-development organisation (CHAI Core: Interventions, Chapel Hill, North Carolina, USA) to design an app that tracks PA levels by logging step counts, and provides users with culturally tailored motivational messages created in partnership with DC CHOC. We named the app 'Step It Up', in reference to the desired outcome of increasing the number of daily steps among participants.
Participants used this mobile phone app alongside a wearable PA tracker, the Fitbit Charge 2 (Fitbit, San Francisco, California, USA), for 20 days. Subsequently, FGs were conducted at a local partnering church to understand participants' experiences using the app to overcome PA barriers and increase PA, described in previous work. ${ }^{38}$ These FG data were used to create a second iteration of the Step It Up app, subject to further user experience interviews and pilot testing. We learnt from one-on-one user experience interviews focused on wire-frame design that participants preferred bold colour schemes, audiovisual supplements such as instructional videos on stretching, and wanted an independent section for goal-setting and tracking progress. Users also felt this app could be used as a replacement or supplement to commercial fitness apps. This feedback was incorporated in a third iteration through a 21-day pilot, leading to development of the Step It Up app for the larger intervention.

\section{Design and overview of Step It Up intervention}

Sequential Multiple-Assignment Randomized Trial design

Our intervention uses a Sequential Multiple-Assignment Randomized Trial (SMART) design, a method for the development of rapid adaptive behavioural interventions. ${ }^{39} 40$ This method rerandomizes patients who have not reached the PA goal of 10000 steps/day after 3 months to more intensive treatment (figure 2). SMART design investigates the impact of intensifying messaging on PA levels among initial non-responders.

\section{Objectives of Step It Up intervention}

The primary objective of this study is to determine if a SMART-designed adaptive mHealth intervention with remote messaging tailored to neighbourhoodenvironment resources (ie, tailored-to-place messaging (TPM)) will increase PA (measured as steps/day) more than standard remote messaging (SRM) (figure 2). This PA intervention will target overweight/obese AA women at risk for cardiometabolic disease with insulin resistance in resource-limited neighbourhoods of the Washington, DC metropolitan area.

A secondary objective is to examine which of four embedded adaptive interventions produces the largest PA increase over the 6-month study period. The four interventions are as follows (figure 2):

Intervention 1

- First 3 months: TPM.

- Second 3 months: TPM+face-to-face coaching.

Intervention 2

- First 3 months: TPM.

- Second 3months: TPM with increased message frequency.

Intervention 3

- First 3 months: SRM.

- Second 3 months: SRM+face-to-face coaching.

Intervention 4

- First 3 months: SRM.

- Second 3 months: TPM. 
Target Population:

- $\mathrm{BMI} \geq 25 \mathrm{~kg} / \mathrm{m}^{2}$

- Insulin resistant

- Resident of target

Washington, D.C. area neighbourhoods

Run-In Period:
If mean PA below
7500 steps/day,
randomised to
Group 1 or 2

2 weeks

2 weeks

\section{Response $=$ 10,000 steps/day?}

* continue using PA monitor

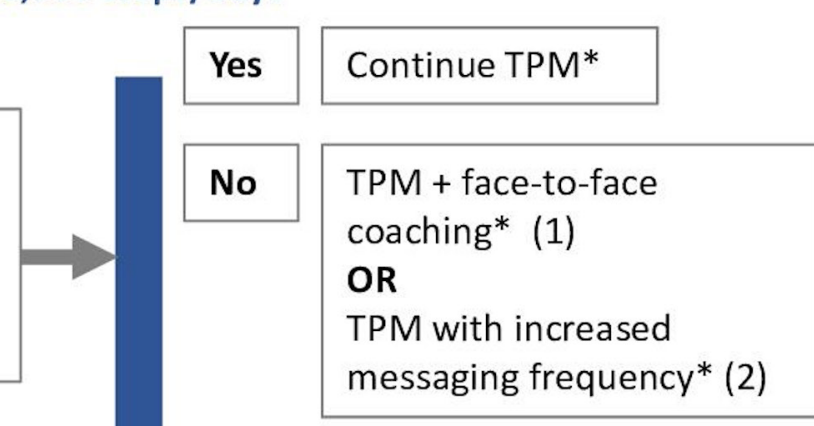

\section{Group 2:}

PA monitor with standard remote messaging (SRM)

\section{Group 1:}

PA monitor with remote tailoredto-place messaging (TPM) messaging frequency* (2)

\section{Yes Continue SRM*}

SRM + face-to-face

coaching* (3)

OR

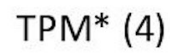

Figure 2 Step It Up: A Sequential, Multiple-Assignment Randomised Trial targeting physical activity (PA) with standard and tailored remote messaging. Four intervention types are as follows: (1) TPM followed by TPM+face-to-face coaching, (2) TPM followed by TPM with increased messaging frequency, (3) SRM followed by SRM+face-to-face coaching, (4) SRM followed by TPM. Patients initially randomised to standard remote messaging (SRM) who do not reach goal of 10000 steps/day by the end of 3 months will be rerandomised to receive tailored-to-place messaging (TPM) or SRM coupled with face-to-face coaching. Similarly, patients initially randomised to TPM who do not reach goal of 10000 steps/day will be rerandomised to TPM supplemented with face-to-face coaching or increased messaging frequency. BMI, body mass index.

Other secondary objectives include evaluating the feasibility of mHealth technology for remote capture of cardiovascular measures, including blood pressure, weight and blood glucose. Intervention effects on clinical markers (table 1) and how behavioural and psychosocial factors mediate the relationship between PA change and cardiovascular health will also be examined. Finally, exploratory analyses will examine the impact of changes in PA levels on metabolic and inflammatory pathways using blood samples collected at baseline, 3 and 6 months.

Table 1 Clinical measures and biological markers

\begin{tabular}{|c|c|}
\hline Measure & Description \\
\hline \multicolumn{2}{|l|}{ Clinical measures } \\
\hline \multicolumn{2}{|l|}{ Cardiovascular health markers } \\
\hline \multicolumn{2}{|c|}{ Biological markers of immune system activation } \\
\hline Immune cell phenotyping & $\begin{array}{l}\text { Flow cytometry-based characterisation of immune cell populations and their receptor } \\
\text { expression profile from flow panels for leucocyte cell populations including neutrophils, } \\
\text { eosinophils, basophils, T-cells, B-cells, NK cells, NKT cells and monocytes }\end{array}$ \\
\hline Biomarker-based profiling & $\begin{array}{l}\text { Cytokine/chemokine }{ }^{75} \text { (TNF } \alpha \text {, IL-6, IL-10, IL-8, IL-1 } \beta \text {, IFN } \alpha \text {, IFN } \gamma \text {, MCP-1, IL-1RA, IL-18), } \\
\text { stress-induced neurotransmitter profiling (dopamine, epinephrine and norepinephrine) and } \\
\text { other biomarkers associated with CVD (VEGF-A, TGF } \beta, T M A O)^{76}\end{array}$ \\
\hline
\end{tabular}

NKT: Natural Killer T cells. TNF $\alpha$ : Tumor Necrosis Factor alpha. VEGF: Vascular Endothelial Growth Factor, TFGbeta: Transforming Growth Factor Beta, and TMAO: Trimethylamine N-Oxide.

BMI, body mass index; CVD, cardiovascular disease; IFN, interferon; IL, interleukin; NK, natural killer; PA, physical activity. 
This study will include (1) cardiometabolic screening and measurements, (2) administration of a detailed survey tailored by community input ${ }^{41}$ and (3) deployment of the Step It Up mobile app and handheld mHealth technology to promote increased PA. ${ }^{38}$ All participants will receive counselling and written information regarding steps to improve their dietary intake in accordance with the Dietary Approach to Stop Hypertension dietary pattern on study enrolment. ${ }^{42}$ Online supplemental table 1 summarises protocol design.

\section{Recruitment of participants}

Inclusion criteria

Overweight or obese (body mass index (BMI) $\geq 25 \mathrm{~kg}$ / $\mathrm{m}^{2}$ ) AA women aged 25-75 years with evidence of insulin resistance based on fasting plasma glucose (100-125 mg/ $\mathrm{dL})$ or haemoglobin A1c (HgAlc $=5.7 \%-6.4 \%)$ living in Washington, DC Wards 5, 7, 8 or Prince George's County, MD are eligible. Participants must have access to a smartphone compatible with the Step It Up mobile app. Participants must be able to provide informed consent independently and speak and read English at or above an eighth grade level.

\section{Exclusion criteria}

Prospective participants with the following characteristics will be excluded: (1) medical condition, including recent unintentional weight loss, which might prohibit safe participation in this intervention; (2) existing selfreported diagnosis of diabetes mellitus; (3) fasting blood glucose $\geq 126 \mathrm{mg} / \mathrm{dL}$ or haemoglobin A1c $\geq 6.5 \%$ without prior diagnosis of diabetes mellitus; (4) heart disease, as indicated by one of the following: history of myocardial infarction, documented disease on coronary angiography, coronary artery stent placement, congestive heart failure or significant structural heart disease; (5) hyperthyroid or hypothyroid by lab screening; (6) physically unable to perform PA for any reason; (7) pregnancy.

\section{Methods of participant recruitment}

Participants will be recruited through flyers and advertisements at partnering churches and community organisations in the target lower-resourced neighbourhoods. We will work with community partners in DC CHOC to promote community members' participation, and encourage participants to invite friends and associates, especially those without current exercise routines. The National Institutes of Health (NIH) Intramural Institutional Review Board (IRB) approved this study and all participants will provide written informed consent (see online supplemental file 1), obtained with trained research staff, prior to participating in this study.

\section{Elements of the Step It Up mHealth PA intervention mHealth app overview}

All participants will receive a password-protected mobile app providing informational modules on the benefits of PA, potential barriers to PA and methods to overcome these barriers. The educational material within these modules is drawn from the Diabetes Prevention Programme curriculum, an evidence-based health behaviour change programme designed for individuals with insulin resistance, like Step It Up participants. ${ }^{43}$ The mobile app is linked to a wearable PA monitor, which assesses steps, sleep duration and heart rate; this data will inform custom daily messages encouraging participants to participate in PA (figure 3). Step goals will be based on a participant's activity; each week, her step goal will be $110 \%$ of the prior week's average daily step count, to a maximum of 10000 steps.
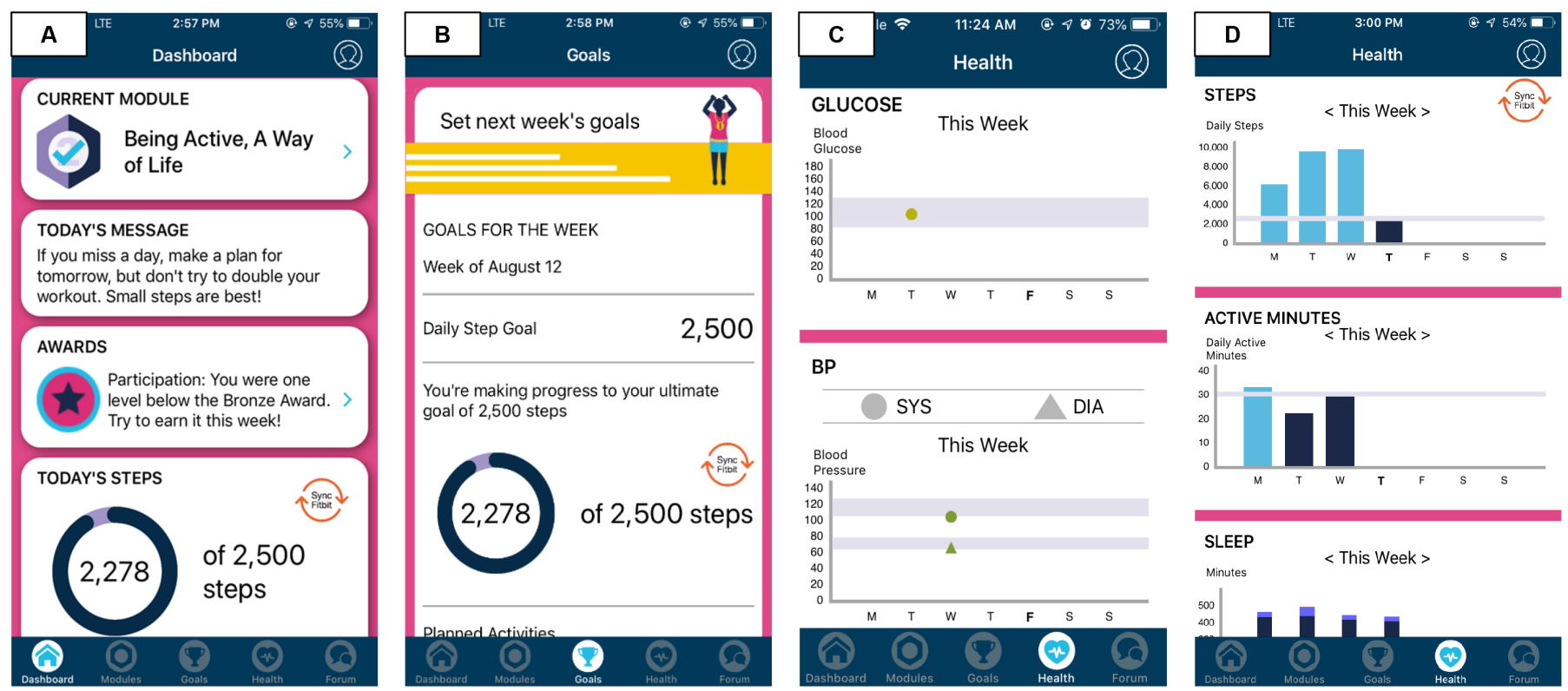

Figure 3 Components of Step It Up app with (A) a dashboard featuring educational modules, daily motivational messages and awards, (B) a goal-setting page and (C, D) graphs to self-monitor physical activity (PA) and health metrics measured by Bluetooth-enabled PA monitor, scale, blood pressure cuff and glucometer. The app also includes a social forum, not pictured. 


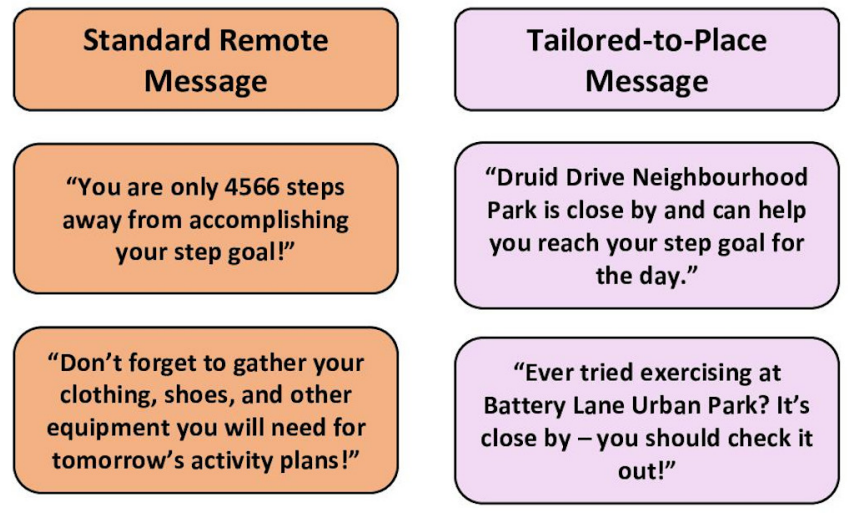

Figure 4 Examples of standard remote messages, which are goal-based and time-based, in comparison to locationbased tailored-to-place messages.

\section{Participant randomisation}

Prior to enrolment, research staff will assign each participant study number to one of the two starting interventions using computer-generated random numbers. The community research coordinator will enrol participants and assign a study number sequentially in order of enrolment. The research team and participants will not be blinded to the intervention or key outcomes.

\section{Standard remote messaging}

Participants randomised to SRM will receive the mHealth app with messages that promote PA by focusing on individual-level factors such as goal-setting and selfefficacy. Daily messaging was developed with input from the community advisory board, DC CHOC. A questionnaire was emailed to DC CHOC to solicit feedback about PA barriers, and members contributed motivational messaging promoting PA for the app. Their suggestions were followed up with phone interviews to further clarify and tailor messaging to AA women (figure 4), as described previously. $^{38}$

\section{Remote TPM}

Participants randomised to TPM will receive the same mHealth app, with different messaging based on the socioecological model (figure 1). This model considers each individual's behaviour to be influenced by a variety of interrelated institutional, environmental and community factors, thereby emphasising the importance of environmental context in behaviour change. TPM will reference certain locations within the participants' neighbourhood environment, in close proximity to her work, home or church, which are suitable for PA (figure 4). As such, although all participants will have access to the same educational modules and goal-setting tools within the mHealth app, the TPM group will also receive messaging tailored to PA resources within each participants' activity space, or geographic region where they spend time. Specifically, our technology will use GPS to determine when a participant comes near a designated PA venue, ${ }^{44}$ and the app will generate a real-time, TPM to notify the participant of this venue and encourage PA.

\section{Geofencing}

TPM uses real-time geospatial location information of the participant and known PA locations within the region studied. The mobile app connects to a registry of public locations suitable for PA, including community recreation centres and parks, in addition to PA locations provided to us by the participants themselves. The registry was created through a detailed search for PA opportunities in the Washington, DC metropolitan area, followed by discussion with the community advisory board. The mobile app sets a geofence, or geographic boundary, in a 0.75 mile (approximately $1200 \mathrm{~m}^{44}$ ) radius around each PA venue. This distance was selected because it represents a $10-15$ min walk. ${ }^{45}{ }^{46}$ When the study participant enters this boundary at a time designated to receive a message, the app will generate a TPM.

\section{PA and sleep monitoring}

To objectively measure PA and sleep duration, each participant will be provided with a commercially available wireless PA and sleep monitoring wristband device (Fitbit Charge 2, Fitbit), and encouraged to wear this device 24 hours/day, except when bathing or immersed in water. This device will enable participants to selfmonitor PA, including active minutes as determined by heart rate, step count and sleep duration for the entirety of the trial. The Fitbit was chosen due to user preference and validity in measuring outcome variables. Prior FG users particularly enjoyed the Fitbit compared with other wearable PA-monitors for its comfort, features and easeof-use. ${ }^{3438}$ The Fitbit is validated to reliably measure step count, heart rate and sleep duration, which are assessed in this study. ${ }^{47-49}$ The Step It Up mobile app will connect with the PA-monitor, allowing participants to track their PA and sleep through the app.

\section{Mobile health tools}

We will provide a subset (up to 25) of participants with a wireless blood pressure monitor, a weight scale and/ or a blood glucose monitor throughout the study period to test the feasibility of using other mHealth tools to remotely monitor cardiometabolic markers. Each device will have capability for wireless data upload to participants' smartphone and the Step It Up app. Participants with these devices will be encouraged to measure blood pressure, weight or blood glucose at least once per week using the device, but device use will be optional and at the discretion of the participant.

\section{Strategies for adherence and retention}

To promote adherence, we adjusted the intervention based on feedback from the community advisory board, the Washington, DC CHNA, and pilot testing. Data will be collected as available in cases of non-adherence. Our community research coordinator is a liaison between community members, study participants and the research 
team, who promotes enrolment and checks in with participants to encourage adherence. Financial compensation for continuous participation in the study and minimal clinic visits for participant convenience are intended to promote retention.

\section{Outcome measures and evaluation}

\section{Survey data}

A survey instrument will be administered with items focusing on health history, access and the behavioural, psychosocial, cultural and perceived environmental factors that may influence the impact of the intervention (see online supplemental table 2) ${ }^{61-67}$ This survey was used in the Washington, DC CHNA, and tailored to the needs of the target community using group-based cognitive interviewing. ${ }^{68}$ Each participant will complete this survey instrument on beginning the study.

\section{Neighbourhood data}

Objective neighbourhood environment measures including Walk Score, Active Neighborhood Checklist and Neighborhood Deprivation Index ${ }^{69}$ are determined based on census data and participant-provided locations for home, work and place of worship (see online supplemental table 2). ${ }^{61-67}$

\section{mHealth data}

Data from Fitbit, including step count, minutes of vigorous activity and sleep time, will be transferred to the Step It Up app. If participants use elective mHealth devices (scale, blood glucose monitor, blood pressure cuff) data will be collected from the associated apps, including weight, blood glucose and blood pressure.

\section{Clinical data}

Participants' health measures related to the study will be collected at clinic visits at baseline, 3-month and 6-month follow-up (table 1). Adverse health events will be monitored and recorded in accordance with IRB protocol. We will also collect blood samples to evaluate effects of PA changes on immune cells involved in CVD and the chronic stress response. Specifically, we will perform flow cytometry panels to (1) characterise the overall immune cell profile, ${ }^{70}(2)$ analyse monocyte subsets and receptor expression (known to be critically involved with atherogenesis) ${ }^{71-73}$ and (3) identify natural killer cell subsets (known to be deactivated with social stress and obesity). ${ }^{74}$ We will profile immunoregulating proteins from participants' serum alongside stress-induced neurotransmitters and other proteins associated with CVD progression (table 1). ${ }^{7075} 76$

\section{Sample size calculation}

Sample size calculations for this intervention are based on determining the main effect of the initial treatment in the adaptive design at $80 \%$ power and for a standardised mean-difference effect size of 0.5 . An equal number in each initial treatment group being compared is assumed.
The sample size formula assumes normality and uses the standard formulae from a Student's t-test. Based on these assumptions, the intervention will require 126 participants. We will recruit 180 residents from the targeted neighbourhoods, of which we assume $30 \%$ will not meet study criteria and/or will be lost to follow-up during the run-in period.

\section{Data analysis plan}

The main objective is to test the primary hypothesis that an adaptive PA intervention with mobile app-based remote TPM will lead to greater PA levels than SRM over a 3-month period. To test this hypothesis, the daily step count from baseline to 6 months postrandomisation will be collected. An algorithm will examine minute-byminute step count data from the PA monitor in blinded fashion to identify days when the PA monitoring device was not worn at all, worn for fewer than 10 hours/day, or was not working; those days will have missing values. ${ }^{77}$

A mean number of steps/day (Y) will be computed for each participant based on the available non-missing days for each week $(T=-1$ to $T=26)$. If a subject is initially randomised to TPM, then $\mathrm{A}=1$. If the subject is randomised to $S R M$, then $A=0$. If a subject with $A=1$ is rerandomised to $\mathrm{TPM}+$ face-to-face messaging, then $\mathrm{B}=1$. If a subject with $A=1$ is rerandomised to TPM+increased messaging frequency, then $B=-1$. If a subject with $A=0$ is rerandomised to $\mathrm{SRM}+$ face-to-face messaging, then $\mathrm{B}=1$. If a subject with $\mathrm{A}=0$ is rerandomised to TPM, then $\mathrm{B}=-1$. Otherwise, any subject not rerandomised has $\mathrm{B}=0$ (see online supplemental table 3).

Classify the subjects' age at enrolment as AGE. We will fit the linear mixed model to the $\mathrm{Y}$ values $\left(\mathrm{Y}_{-1}, \mathrm{Y}_{0}, \mathrm{Y}_{1}, \ldots\right.$, $\mathrm{Y}_{26}$ ) using the function LME from the package NLME in R package V.3.1 ${ }^{78}$ :

$$
\begin{aligned}
& \mathrm{E}\left[\mathrm{Y}_{\mathrm{T}} \mathrm{IT}, \mathrm{A}, \mathrm{B}, \mathrm{AGE}\right]=\beta_{0}+\beta_{\mathrm{AGE}} \cdot \mathrm{AGE}+\sum_{\mathrm{t}=-1}^{26} \beta_{\mathrm{T}=\mathrm{t}}^{\mathrm{l}}(\mathrm{T}=\mathrm{t})+ \\
& \sum_{\mathrm{t}=1}^{26} \beta_{\mathrm{A}: \mathrm{T}=\mathrm{t}} \cdot \mathrm{A} \cdot \mathrm{I}_{(\mathrm{T}=\mathrm{t})} \cdot \mathrm{A} \cdot \mathrm{I}_{\mathrm{T}=\mathrm{t}}+\sum_{\mathrm{t}=14}^{26} \beta_{\mathrm{B}: \mathrm{A}=0, \mathrm{~T}=\mathrm{t}} \cdot \mathrm{B} \cdot[1-\mathrm{A}] \\
& \cdot \mathrm{I}_{(\mathrm{T}=\mathrm{t})}+\sum_{\mathrm{t}=14}^{26} \beta_{\mathrm{B}: \mathrm{A}=1, \mathrm{~T}=\mathrm{t}} \cdot \mathrm{B} \cdot \mathrm{A} \cdot \mathrm{I}_{(\mathrm{T}=\mathrm{t})}+\sum_{\mathrm{t}=14}^{26} \beta_{\mathrm{NR}: \mathrm{A}=0, \mathrm{~T}=\mathrm{t}}+ \\
& \cdot[1-\mathrm{A}] \cdot \mathrm{I}_{(\mathrm{B}=0)} \cdot \mathrm{I}_{(\mathrm{T}=\mathrm{t})} \sum_{\mathrm{t}=14}^{26} \beta_{\mathrm{NR}: \mathrm{A}=1, \mathrm{~T}=\mathrm{t}} \cdot \mathrm{A} \cdot \mathrm{I}_{(\mathrm{B}=0)} \cdot \mathrm{I}_{(\mathrm{T}=\mathrm{t})}
\end{aligned}
$$

The effect of treatment for the primary comparison of TPM versus SRM is based on the fitted value of $\beta_{\mathrm{A}: \mathrm{T}=13}$, the predicted model difference between the randomised arms will be at 13 weeks. We further hypothesise that, among TPM non-responders, face-to-face coaching will increase PA more than will increased messaging frequency. This comparison at 26 weeks is based on the fitted model as $2 \cdot \beta_{\mathrm{B}: \mathrm{A}=1, \mathrm{~T}=26}$. Further, we hypothesise that among SRM non-responders, SRM with face-to-face coaching will increase PA over TPM alone, based on the fitted model as 2. $\beta_{\mathrm{B}: \mathrm{A}=0 \mathrm{~T}=26}$. Finally, at 26 weeks, contrasts will be constructed to estimate the differential effects of the four embedded treatment regime strategies. This comparison 
of embedded adaptive interventions is similar to the methods of Nahum-Shani et al. ${ }^{79}$

Sensitivity analyses will be performed to test for significant interactions between age and treatment, and if the relationship between $\mathrm{Y}$, age and other covariates is non-linear. The distribution of cardiovascular health factors between randomisation groups using the $\chi^{2}$ test will be compared. We will also compare dietary intake and self-reported PA by psychosocial and environmental factors, including levels of social support, social isolation, perceived health, perceived spiritual locus of control and objective/perceived neighbourhood environment, using analysis of variance or Student's t-test for continuous variables and $\chi^{2}$ testing for categorical variables. ${ }^{54-65}$

\section{Patient and public involvement}

The community advisory board, DC CHOC, consists of community leaders and potential participants. DC CHOC assisted in development of the research question, outcome measures and assessed the burden of the intervention through participation in DC CHNA. Community members (potential patients/participants) assisted with study design through FGs, iterative testing and reviewing intervention elements. DC CHOC advertised the study through partnering community organisations, and participants will be encouraged to recruit peers. Through the iterative process of developing the trial, we gathered feedback on the burden of the study from participants. Results and conclusions will be disseminated to participants and community members via a quarterly newsletter and presentations with DC CHOC.

\section{DISCUSSION}

This novel mHealth PA intervention serves to better understand and reduce cardiovascular health disparities among AA women living in resource-limited communities.

\section{Strengths}

This intervention reaches an understudied population often left out from traditional healthcare models. The multilevel approach tailors the intervention to women living in and near Washington, DC by targeting the individual and neighbourhood levels; the goal is to help women shape perceptions about existing community resources and use their environment to increase PA. ${ }^{80}$ We established community partnerships and built on a strong foundation of preliminary community-based participatory research studies to ensure trust and collaboration with the community, which is essential for acceptance and success of the community intervention. We also leverage mHealth technology and Bluetooth-enabled devices to reach participants outside the scope of traditional medical settings. The bio-behavioural intervention design focuses on both clinical measures and biological mediators of cardiovascular health. Finally, the statistical approach for this study is unique from analyses of prior
SMART-designed interventions and leverages the large quantity of PA data logged from the Fitbit device.

\section{Limitations}

Statistical power of the study was determined for the primary objective, to evaluate effects of TPM compared with SRM on PA, when delivered through mHealth technology. This study may be under-powered to evaluate changes in cardiovascular risk markers, but exploratory analyses will look for trends. If successful, we will consider a larger study powered to evaluate the impact of the technology-based intervention on BMI or waist-to-hip ratio as outcomes. We will also consider extending this study to other at-risk populations in Washington, DC, including AA men and other racial/ethnic groups, and to additional resource-limited, urban areas in the USA.

\section{Ethics and dissemination}

We submitted this proposed protocol to the National Heart, Lung and Blood Institute (NHLBI) IRB and received full approval for this study. All data will be stored securely, with password protection and encryption, in the electronic health system at the NIH, and will be shared only as needed with approved research personnel. All personal health information in the mHealth app, clinical information, survey and interview data will be de-identified. All biological specimens will be stored within a Food and Drug Administration-approved biospecimen tracking inventory system. Future use of biospecimens not defined in the research protocol will occur only after IRB review and approval, and participants may refuse to allow future use. This study is scheduled to start in 2021 with plans for completion by 2023. The COVID-19 pandemic, which likely alters activity space due to quarantine restrictions, has delayed enrolment. Further, geospatial data collected during the prepandemic pilot study can be compared with geospatial data in the protocol, to assess impact of the pandemic on activity space. Patients who suffer harm from trial participation will be treated according to best practices under the NIH Clinical Center. This study is lowrisk, requiring no data-monitoring committee. The Principal Investigator will monitor accrual and safety data. The protocol will be reviewed and monitored annually by the IRB and the NHLBI Office of the Clinical Director's Protocol Audit Team, with any amendments requiring IRB approval.

Results of this study will be analysed by the research team per IRB policy. Once publicly available, results and conclusions of the work will be shared with community members.

\section{Author affiliations}

${ }^{1}$ Social Determinants of Obesity and Cardiovascular Risk Laboratory, Cardiovascular Branch, Division of Intramural Research, National Heart, Lung, and Blood Institute, National Institutes of Health, Bethesda, Maryland, USA

${ }^{2}$ Office of Biostatistics Research, Division of Cardiovascular Sciences, National Heart, Lung, and Blood Institute, National Institutes of Health, Bethesda, Maryland, USA 
${ }^{3}$ University of North Carolina, Nutrition Obesity Research Center, Lineberger Comprehensive Cancer Center, Connected Health Applications and Interventions (CHAl) Core, Chapel Hill, North Carolina, USA

${ }^{4}$ Department of Psychological and Brain Sciences, George Washington University, Washington, District of Columbia, USA

${ }^{5}$ Health Ministry, Pilgrim Rest Baptist Church, Washington, DC, USA

${ }^{6}$ National Institutes of Health Clinical Center, Nursing Department, Bethesda,

Maryland, USA

${ }^{7}$ Intramural Research Program, National Institute on Minority Health and Health Disparities, National Institutes of Health, Bethesda, Maryland, USA

\section{Twitter Nithya P Vijayakumar @nithyapv_}

Acknowledgements We acknowledge DC CHOC and all of the participants who have made this community-based research possible. This work was supported by the Division of Intramural Research of the National Heart, Lung, and Blood Institute and the Intramural Research Program of the National Institute on Minority Health and Health Disparities (National Institutes of Health). KT is supported by the 2019 Lenfant Biomedical Fellowship Award from the National Heart, Lung, and Blood Institute. Special thanks are given to RM and his staff (Dr Saxena and Dr Dagur) at the NHLBI Flow Cytometry Core. This research was also made possible by the National Institutes of Health (NIH) Medical Research Scholars Programme, a publicprivate partnership supported jointly by the $\mathrm{NIH}$ and generous contributions to the Foundation for the NIH from the Doris Duke Charitable Foundation, Genentech, the American Association for Dental Research, the Colgate-Palmolive Company, Elsevier, alumni of the student research programmes and other supporters via contributions to the Foundation for the National Institutes of Health. Additionally, this work was supported in part by the University of North Carolina at Chapel Hill's Connected Health Applications \& Interventions Core (CHAl Core) through a grant from NIH (DK056350) to the University of North Carolina Nutrition Obesity Research Center AND/OR from NCl (P30-CA16086) to the Lineberger Comprehensive Cancer Center.

Contributors TP-W conceptualised the study. KT and NPV drafted the manuscript. $\mathrm{KC}, \mathrm{SJN}, \mathrm{YB}, \mathrm{CAG}-\mathrm{H}$ and BSC assisted in study design and revised the manuscript. VMM recruited participants and enrolled participants in this protocol and preceding works cited. TMPW designed the use of clinical data. YB, CAG-H, RI and BST performed all immune cell characterisation and biomarker profiling experiments. GRW, SL, PJM, TD and MD-B provided critical feedback on the concept and content KGT, SG, RM and JZ developed the mHealth app. MA, JNC and SEC were involved in prior work for development of initial studies for the protocol and prior work cited. JFT contributed to statistical analyses and developed the unique statistical method applied. All authors contributed to study design, reviewed and approved the final draft of the manuscript.

Funding Funding for the Social Determinants of Obesity and Cardiovascular Risk Laboratory is provided through the Division of Intramural Research (DIR) of the NHLBI of the NIH, and through the Intramural Research Programme of the NIMHD of the NIH, under grant numbers ZIA-HL006168 and ZIA-MD000010.

Disclaimer The views of the present study are those of the authors and do not necessarily represent the views of the National Heart, Lung, and Blood Institute (NHLBI), the National Institute on Minority Health and Health Disparities (NIMHD), the National Institutes of Health $(\mathrm{NIH})$ or the US Department of Health and Human Services.

Competing interests None declared.

Patient consent for publication Not required.

Provenance and peer review Not commissioned; externally peer reviewed.

Supplemental material This content has been supplied by the author(s). It has not been vetted by BMJ Publishing Group Limited (BMJ) and may not have been peer-reviewed. Any opinions or recommendations discussed are solely those of the author(s) and are not endorsed by BMJ. BMJ disclaims all liability and responsibility arising from any reliance placed on the content. Where the content includes any translated material, BMJ does not warrant the accuracy and reliability of the translations (including but not limited to local regulations, clinical guidelines, terminology, drug names and drug dosages), and is not responsible for any error and/or omissions arising from translation and adaptation or otherwise.

Open access This is an open access article distributed in accordance with the Creative Commons Attribution Non Commercial (CC BY-NC 4.0) license, which permits others to distribute, remix, adapt, build upon this work non-commercially, and license their derivative works on different terms, provided the original work is properly cited, appropriate credit is given, any changes made indicated, and the use is non-commercial. See: http://creativecommons.org/licenses/by-nc/4.0/.

ORCID iDs

Kosuke Tamura http://orcid.org/0000-0002-4920-2856

Nithya P Vijayakumar http://orcid.org/0000-0002-8231-5713

Tiffany M Powell-Wiley http://orcid.org/0000-0001-9488-4131

\section{REFERENCES}

1 Chaix B. Mobile sensing in environmental health and neighborhood research. Annu Rev Public Health 2018;39:367-84.

2 Piwek L, Ellis DA, Andrews S, et al. The rise of consumer health Wearables: promises and barriers. PLoS Med 2016;13:e1001953.

3 Melton BF, Buman MP, Vogel RL, et al. Wearable devices to improve physical activity and sleep: a randomized controlled trial of collegeaged African American women. J Black Stud 2016;47:610-25.

4 Dean DAL, Griffith DM, McKissic SA, et al. Men on the MoveNashville: feasibility and acceptability of a Technology-Enhanced physical activity pilot intervention for overweight and obese middle and older age African American men. Am J Mens Health 2018;12:798-811.

5 Lewis ZH, Lyons EJ, Jarvis JM, et al. Using an electronic activity monitor system as an intervention modality: a systematic review. BMC Public Health 2015;15:585.

6 Burke LE, Ma J, Azar KMJ, et al. Current science on consumer use of mobile health for cardiovascular disease prevention: a scientific statement from the American heart association. Circulation 2015;132:1157-213.

7 Bort-Roig J, Gilson ND, Puig-Ribera A, et al. Measuring and influencing physical activity with smartphone technology: a systematic review. Sports Med 2014;44:671-86.

8 Puig-Ribera A, Bort-Roig J, González-Suárez AM, et al. Patterns of impact resulting from a 'sit less, move more' web-based program in sedentary office employees. PLoS One 2015;10:e0122474.

9 Bort-Roig J, Martin M, Puig-Ribera A, et al. Uptake and factors that influence the use of 'sit less, move more' occupational intervention strategies in Spanish office employees. Int J Behav Nutr Phys Act 2014;11:152.

10 Carroll JK, Moorhead A, Bond R, et al. Who uses mobile phone health Apps and does use matter? a secondary data analytics approach. J Med Internet Res 2017;19:1.

11 Bland V, Sharma M. Physical activity interventions in African American women: a systematic review. Health Promot Perspect 2017;7:52-9.

12 Leach HJ, O'Connor DP, Simpson RJ, et al. An exploratory decision tree analysis to predict cardiovascular disease risk in African American women. Health Psychol 2016;35:397-402.

13 Carnethon MR, Pu J, Howard G, et al. Cardiovascular health in African Americans: a scientific statement from the American heart association. Circulation 2017;136:e393-423.

14 Lewis BA, Napolitano MA, Buman MP, et al. Future directions in physical activity intervention research: expanding our focus to sedentary behaviors, technology, and dissemination. J Behav Med 2017:40:112-26.

15 Whitt-Glover MC, Keith NR, Ceaser TG, et al. A systematic review of physical activity interventions among African American adults: evidence from 2009 to 2013. Obes Rev 2014;15 Suppl 4:125-45.

16 National Center for Health Statistics. Summary Health Statistics: National Health Interview Survey, 2018. Available: https://ftp.cdc. gov/pub/Health_Statistics/NCHS/NHIS/SHS/2018_SHS_Table_A-14. pdf

17 Rhodes RE, Zhang R, Zhang C-Q. Direct and indirect relationships between the built environment and individual-level perceptions of physical activity: a systematic review. Ann Behav Med 2020;54:495-509.

18 Agurs-Collins T, Persky S, Paskett ED, et al. Designing and assessing multilevel interventions to improve minority health and reduce health disparities. Am J Public Health 2019;109:S86-93.

19 Knapp M, Gustat J, Darensbourg R, et al. The relationships between park quality, park usage, and levels of physical activity in lowincome, African American neighborhoods. Int J Environ Res Public Health 2018;16. doi:10.3390/ijerph16010085. [Epub ahead of print: 3012 2018].

20 Troped PJ, Wilson JS, Matthews CE, et al. The built environment and location-based physical activity. Am J Prev Med 2010;38(4):429-38. S0749-3797(10)00012-7 [pii] (published Online First: 2010/03/24).

21 Winters M, Barnes R, Venners S, et al. Older adults' outdoor walking and the built environment: does income matter? BMC Public Health 2015; $15: 876$ 
22 King WC, Belle SH, Brach JS, et al. Objective measures of neighborhood environment and physical activity in older women. Am J Prev Med 2005;28:461-9. doi:10.1016/j.amepre.2005.02.001

23 Joseph RP, Ainsworth BE, Keller C, et al. Barriers to physical activity among African American women: an integrative review of the literature. Women Health 2015;55:679-99.

24 Gordon-Larsen P, Nelson MC, Page P, et al. Inequality in the built environment underlies key health disparities in physical activity and obesity. Pediatrics 2006;117:417-24. doi:10.1542/peds.2005-0058

25 Thomas S, Yingling L, Adu-Brimpong J, et al. Mobile health technology can objectively capture physical activity (PA) targets among African-American women within resource-limited communities - the Washington, D.C. cardiovascular health and needs assessment. J Racial Ethn Health Disparities 2017;4:876-83. doi:10.1007/s40615-016-0290-4

26 Yingling LR, Mitchell V, Ayers CR, et al. Adherence with physical activity monitoring wearable devices in a community-based population: observations from the Washington, D. C., Cardiovascular Health and Needs Assessment. Transl Behav Med 2017;7:719-30.

27 District of Columbia Government, 2019District of Columbia Behavioral Risk Factor Surveillance System (BRFSS): 2017 Annual Health Report. Available: https://dchealth.dc.gov/publication/brfssannual-report-2017

28 Institute on Metropolitan Opportunity. American neighborhood change in the 21st century. 36. Minneapolis, MN: University of Minnesota Law School, 2019.

29 U.S. Census Bureau. Quick Facts, Prince George's County, Maryland, 2020. Available: https://www.census.gov/quickfacts/fact/ table/princegeorgescountymaryland/RHI225218\#RHI225218

30 Smith R. Physical activity and gym access by neighborhood in D.C: D.C. policy center, 2017. Available: https://www.dcpolicycenter.org/ publications/gym-deserts/

31 Ceasar J, Peters-Lawrence MH, Mitchell V, et al. The communication, awareness, relationships and Empowerment (C.A.R.E.) model: an effective tool for engaging urban communities in community-based participatory research. Int $J$ Environ Res Public Health 2017;14. doi:10.3390/ijerph14111422. [Epub ahead of print: $21112017]$

32 Agyemang P, Powell-Wiley TM. Obesity and black women: special considerations related to genesis and therapeutic approaches. Curr Cardiovasc Risk Rep 2013;7:378-86.

33 Wallen GR, Saygbe JN, Brooks AT, et al. Engaging community leaders in the development of a cardiovascular health behavior survey using focus Group-Based cognitive interviewing. Health Services Insights 2017;10:117863291770112.

34 Yingling LR, Brooks AT, Wallen GR, et al. Community engagement to optimize the use of web-based and wearable technology in a cardiovascular health and needs assessment study: a mixed methods approach. JMIR mHealth uHealth 2016;4:e38-55.

35 Tigbe WW, Granat MH, Sattar N, et al. Time spent in sedentary posture is associated with waist circumference and cardiovascular risk. Int J Obes 2017;41:689-96.

36 Newton RL, Han H, Johnson WD, et al. Steps/day and metabolic syndrome in African American adults: the Jackson heart study. Prev Med 2013;57:855-9. doi:10.1016/j.ypmed.2013.09.018

37 Lee I-M, Shiroma EJ, Kamada M, et al. Association of step volume and intensity with all-cause mortality in older women. JAMA Intern Med 2019. doi:10.1001/jamainternmed.2019.0899. [Epub ahead of print: 29 May 2019].

38 Ceasar JN, Claudel SE, Andrews MR, et al. Community engagement in the development of an mHealth-enabled physical activity and cardiovascular health intervention (Step It Up): pilot focus group study. JMIR Form Res 2019;3:e10944.

39 Almirall D, Nahum-Shani I, Sherwood NE, et al. Introduction to SMART designs for the development of adaptive interventions: with application to weight loss research. Trans/ Behav Med 2014;4:260-74

40 Collins LM, Murphy SA, Strecher V. The multiphase optimization strategy (MOST) and the sequential multiple assignment randomized trial (SMART): new methods for more potent eHealth interventions. Am J Prev Med 2007;32:S112-8.

41 Shuger SL, Barry VW, Sui X, et al. Electronic feedback in a dietand physical activity-based lifestyle intervention for weight loss: a randomized controlled trial. Int J Behav Nutr Phys Act 2011;8:41.

42 Karanja NM, Obarzanek E, Lin PH, et al. Descriptive characteristics of the dietary patterns used in the dietary approaches to stop hypertension trial. DASH Collaborative Research Group. J Am Diet Assoc 1999;99:S19-27.

43 Knowler WC, Barrett-Connor E, Fowler SE, et al. Reduction in the incidence of type 2 diabetes with lifestyle intervention or metformin. N Engl J Med 2002;346:393-403.
44 Troped PJ, Starnes HA, Puett RC, et al. Relationships between the built environment and walking and weight status among older women in three U.S. states. J Aging Phys Act 2014;22:114-25.

45 Centers for Disease Control and Prevention (CDC). Cdc vital signs: more people walk to better health centers for disease control and prevention website, 2013. Available: https://www.cdc.gov/vitalsigns/ walking/

46 Powell-Wiley TM, Wong MS, Adu-Brimpong J, et al. Simulating the Impact of Crime on African American Women's Physical Activity and Obesity. Obesity 2017;25:2149-55.

47 de Zambotti M, Goldstone A, Claudatos S, et al. A validation study of Fitbit charge $2^{\mathrm{TM}}$ compared with polysomnography in adults. Chronobiol Int 2018;35:465-76.

48 Nelson BW, Allen NB. Accuracy of consumer wearable heart rate measurement during an ecologically valid 24-hour period: Intraindividual validation study. JMIR Mhealth Uhealth 2019;7:e10828

49 Tedesco S, Sica M, Ancillao A, et al. Validity evaluation of the Fitbit Charge2 and the Garmin vivosmart HR+ in free-living environments in an older adult cohort. JMIR Mhealth Uhealth 2019;7:e13084.

50 Centers for Disease Control and Prevention. Behavioral risk factor surveillance system survey questionnaire, 2011. Available: http:// www.cdc.gov/brfss/questionnaires/english.htm

51 National Cancer Institute. Dietary screener questionnaire (DSQ) in the NHANES 2009-10. Available: http://epi.grants.cancer.gov/nhanes/ dietscreen/questionnaires.html [Accessed August 8, 2020].

52 Centers for Disease Control and Prevention, National Center for Health Statistics (NCHS). National health and nutrition examination survey weight history questionnaire, 2011. Available: http://www.cdc. gov/nchs/data/nhanes/nhanes_11_12/whq.pdf

53 Harris CV, Bradlyn AS, Coffman J, et al. BMI-based body size guides for women and men: development and validation of a novel pictorial method to assess weight-related concepts. Int J Obes 2008;32:336-42.

54 Russell D, Peplau LA, Cutrona CE. The revised UCLA Loneliness Scale: concurrent and discriminant validity evidence. J Pers Soc Psychol 1980;39:472-80.

55 Eaton WW, Muntaner C, Smith C, et al. Center for epidemiologic studies depression scale: review and revision (CESD and CESD-R). In: Maruish ME, ed. The use of psychological testing for treatment planning and outcomes assessment. 3rd ed. Mahwah, NJ: Lawrence Erlbaum Associates Publishers, 2004: 363-77.

56 Cohen S, Kamarck T, Mermelstein R. A global measure of perceived stress. J Health Soc Behav 1983;24:385-96.

57 Brondolo E, Kelly KP, Coakley V, et al. The perceived ethnic discrimination questionnaire: development and preliminary validation of a community Version1. J App/ Soc Psychol 2005;35:335-65.

58 Turner RJ, Wheaton B, Lloyd DA. The epidemiology of social stress. Am Sociol Rev 1995;60:104-25.

59 Buysse DJ, Reynolds CF, Monk TH, et al. The Pittsburgh sleep quality index: a new instrument for psychiatric practice and research. Psychiatry Res 1989;28:193-213.

60 Edinger JD, Sampson WS. A primary care "friendly" cognitive behavioral insomnia therapy. Sleep 2003;26:177-82.

61 Sampson RJ, Raudenbush SW, Earls F. Neighborhoods and violent crime: a multilevel study of collective efficacy. Science 1997;277:918-24.

62 Powell-Wiley TM, Ayers CR, de Lemos JA, et al. Relationship between perceptions about neighborhood environment and prevalent obesity: data from the Dallas heart study. Obesity 2013;21:E14-21.

63 Hoehner CM, Ivy A, Ramirez LKB, et al. Active neighborhood checklist: a user-friendly and reliable tool for assessing activity friendliness. Am J Health Promot 2007;21:534-7.

64 Walk Score. Walk score, 2020. Available: walkscore.com

65 Holt CL, Clark EM, Kreuter MW, et al. Spiritual health locus of control and breast cancer beliefs among urban African American women. Health Psychol 2003;22:294-9.

66 Pew Research Center. Mobile fact sheet: pew research center, 2019. Available: https://www.pewresearch.org/internet/fact-sheet/mobile/\# find-out-more

67 Bunz U. The computer-email-web (CEW) fluency scale-development and validation. Int J Hum Comput Interact 2004;17:479-506.

68 Wallen GR, Saygbe JN, Brooks AT, et al. Engaging community leaders in the development of a cardiovascular health behavior survey using focus group-based cognitive interviewing. Health Serv Insights 2017;10:117863291770112.

69 Powell-Wiley TM, Gebreab SY, Claudel SE, et al. The relationship between neighborhood socioeconomic deprivation and telomere length: the 1999-2002 National health and nutrition examination survey. SSM Popul Health 2020;10:100517. 
70 Baumer Y, Gutierrez-Huerta CA, Saxena A, et al. Immune cell phenotyping in low blood volumes for assessment of cardiovascular disease risk, development, and progression: a pilot study. J Trans/ Med 2020;18:29.

71 Deshmane SL, Kremlev S, Amini S, et al. Monocyte chemoattractant protein-1 (MCP-1): an overview. J Interferon Cytokine Res 2009;29:313-26.

72 Gautier EL, Jakubzick C, Randolph GJ. Regulation of the migration and survival of monocyte subsets by chemokine receptors and its relevance to atherosclerosis. Arterioscler Thromb Vasc Biol 2009;29:1412-8.

73 Snyder-Mackler N, Sanz J, Kohn JN, et al. Social status alters immune regulation and response to infection in macaques. Science 2016;354:1041-5.

74 Nahrendorf M, Swirski FK. Cholesterol, CCR2, and monocyte phenotypes in atherosclerosis. Eur Heart $J$ 2017;38:1594-6.

75 Jalbert E, Crawford TQ, D'Antoni ML, et al. II-1B enriched monocytes mount massive IL-6 responses to common inflammatory triggers among chronically HIV-1 infected adults on stable anti-retrovira therapy at risk for cardiovascular disease. PLoS One 2013;8:e75500.

76 Hilding A, Brismar K, Degerblad M, et al. Altered relation between circulating levels of insulin-like growth factor-binding protein- 1 and insulin in growth hormone-deficient patients and insulin-dependent diabetic patients compared to that in healthy subjects. $J$ Clin Endocrinol Metab 1995;80:2646-52.

77 Claudel SE, Tamura K, Troendle JF, et al. Comparing methods to identify wear time intervals for physical activity with the Fitbit charge 2. J Aging Phys Act 2020;Ahead of print.

78 Pinheiro J, Bates D, DebRoy S. nIme: linear and nonlinear mixed effects models $2020 \mathrm{https}$ ///CRAN.R-project.org/package=nlme

79 Nahum-Shani I, Qian M, Almirall D, et al. Experimental design and primary data analysis methods for comparing adaptive interventions. Psychol Methods 2012:17:457-77.

80 Bakken S, Marden S, Arteaga SS, et al. Behavioral interventions using consumer information technology as tools to advance health equity. Am J Public Health 2019;109:S79-85. 